\title{
'iQuiero cambiar a mi hijo de grupo!'. Factores explicativos de la eficiencia técnica de los colegios en España
}

Núm. 2 (2014), pp. 79-109.

Daniel Santín ${ }^{1}$

Gabriela Sicilia $^{2}$

Recibido: Enero, 2014

Aceptado: Febrero, 2014

JEL Clasif: JEL-class: $121, C 14, H 52$.

\footnotetext{
${ }^{1}$ Departamento de Economía Aplicada VI. Universidad Complutense de Madrid. dsantin@ccee.ucm.es

${ }^{2}$ Departamento de Economía Aplicada VI. Universidad Complutense de Madrid. gabriels@ucm.es
} 


\begin{abstract}
The aim of this research is to identify the main drivers of Spanish primary school efficiency. We are particularly interested in identifying which variables could be influenced by the design of public policies in order to improve academic outcomes with the current resource allocation. To do this, we perform a two-stage semiparametric model using the "Evaluación General de Diagnóstico 20o9" database from the Spanish Ministry of Education. In the first stage, we use Data Envelopment Analysis (DEA) to estimate efficiency scores, which are then regressed on school and student contextual variables. The results show that, on average, Spanish schools can improve their academic outcomes in $12 \%$. Regarding the drivers of this inefficiency, our findings suggest that education policy should focus on supporting students with high risk of repetition at an early age, encouraging daily extracurricular reading, promoting classroom teaching techniques involving more individual work by students and less exposure time by teachers and seeking a greater involvement of parents in their children's education. Finally, the results evidence that when two groups within the same primary school are compared, in 35.5\% (13.5\%) of them the teacher and teaching methods explain five to ten efficiency points (more than ten efficiency points). Therefore, in addition to measuring the schools efficiency level it would be necessary to perform schools' systematic evaluations at the classroom level in order to control the efficiency and equity of the system.
\end{abstract}

Key Words: Primary education, technical efficiency, DEA, bootstrap.

\title{
Resumen
}

Este trabajo tiene como objeto identificar los principales factores determinantes de la eficiencia de la Educación Primaria en España. En particular, interesa identificar aquellas variables sobre las cuales se podría influir a través del diseño de políticas públicas, con el fin de mejorar los resultados alcanzados sin necesidad de incrementar los recursos disponibles. Para ello, se estima un modelo semi-paramétrico en dos etapas, a partir de la información de la Evaluación General de Diagnóstico 2009 del Ministerio de Educación, Cultura y Deporte. En una primera etapa, se estima el nivel de eficiencia de cada uno de los grupos de alumnos de los centros educativos evaluados a través de un modelo DEA (Data Envelopment Analysis), y posteriormente se regresan los índices estimados sobre diversas variables educativas asociadas a los colegios y a los 
‘Quiero cambiar a mi hijo de grupo!’. Factores explicativos de la eficiencia técnica de los colegios en España

alumnos. Los resultados del estudio evidencian que, en media, los colegios tendrían un margen potencial para mejorar un $12 \%$ sus resultados. En cuanto a las causas que explican esta ineficiencia los resultados muestran que la política educativa debería centrar sus esfuerzos en brindar apoyo a los alumnos con mayor riesgo de repetición a edades tempranas, fomentar la lectura diaria extracurricular, promover técnicas de enseñanza en el aula que impliquen más trabajo individual por parte de los alumnos y menos tiempo de exposición por parte de los profesores y en buscar mayor compromiso de los padres en la educación de sus hijos. Finalmente, los resultados evidencian que cuando se comparan dos grupos dentro de un mismo centro de primaria en un $35,5 \%$ $(13,5 \%)$ de colegios el profesor y sus métodos docentes explican entre cinco y diez puntos (más de diez puntos) de eficiencia. Es por ello que además de medir la eficiencia de los centros resulta clave llamar la atención sobre la necesidad de realizar evaluaciones sistemáticas de los centros a nivel de grupo, para controlar la eficiencia y la equidad del Sistema³.

Palabras clave: Educación Primaria, Eficiencia técnica, DEA, bootstrap.

\footnotetext{
3 Este trabajo ha sido elaborado dentro del Proyecto de Investigación "La medición de la eficiencia de la educación primaria y de sus determinantes en España y en la Unión Europea” financiado por la Fundación Ramón Areces a la cual los autores agradecen su apoyo. Asimismo, los autores agradecen los comentarios recibidos durante el proceso de evaluación de esta investigación.
} 


\section{Introducción}

La cantidad de años de educación que un individuo recibe a lo largo de su vida junto a la calidad de la misma son determinantes de su bienestar futuro. Individuos más y mejor formados disponen en media de mejor acceso y adaptación en el mercado laboral y mayor remuneración, todo ello como consecuencia de la mayor productividad que les reporta su mejor nivel educativo. Por este motivo, la inversión que un país realiza en educación de calidad es fundamental para asegurar el desarrollo y el crecimiento económico (Barro, 2001; Hanushek y Kimko, 2000; Hanushek y Woessman, 2008; De la Fuente, 2011). Por tanto, no resulta sorprendente que el gasto educativo sea uno de los principales rubros del presupuesto público en la mayor parte de los estados modernos. En efecto, la tendencia en la gran mayoría de los países de la OCDE en la última década ha sido incrementar la inversión presupuestaria destinada a educación, proceso que se ha detenido e incluso revertido en algunos países como consecuencia de la actual situación económica.

En particular, España ha realizado un enorme esfuerzo en la década pasada para incrementar los niveles de inversión en materia educativa. Así, mientras que en el año 2001 el porcentaje del PIB destinado a educación era del 4,24\%, en el año 2010 dicha cifra representaba casi el $5 \%$ del PIB (Eurostat), lo cual constituye un incremento del $17 \%$, cifra considerablemente superior al incremento promedio de la UE-27 que en el mismo período fue de $9 \%$. No obstante, este esfuerzo en materia presupuestaria no se ha visto acompañado de reformas educativas exitosas y políticas adecuadas que hayan mejorado los logros educativos. España continua ocupando uno de los peores lugares de la lista de países de la OCDE en resultados educativos, según evidencian diversos programas de evaluación internacionales como las últimas oleadas del informe PISA (Programme for International Student Assessment) o los recientes resultados en el informe TIMSS (Trends en International Mathematics and Science Study) y PIRLS (Progress in International Reading Literacy Study) 2011. Además, España continua presentando en 2011 una tasa de abandono educativo del 26,5\% muy superior al 13,5\% de la media europea (UE-27). Si bien se ha querido presentar este resultado como una cuantiosa mejoría respecto a los últimos años (en 2001 era del 32\%), lo cierto es que dentro de la UE-27 sólo Malta presentaba en 2011 un resultado peor.

La publicación de estos niveles de rendimiento y resultados educativos provoca que las autoridades muestren su enorme desconcierto cada vez que se publican nuevas 
‘Quiero cambiar a mi hijo de grupo!’. Factores explicativos de la eficiencia técnica de los colegios en España

cifras. El hecho de obtener unos resultados mediocres en comparación con el gasto educativo invertido hacen que surjan interrogantes acerca de cómo cambiar esta tendencia. Con cada cambio de gobierno en España se ha ido produciendo un cambio de la Ley Educativa, lo cual pone de manifiesto la falta de consenso que existe a nivel nacional sobre cómo mejorar la educación, si bien todos los actores coinciden en que es un aspecto clave para el crecimiento y el desarrollo económico futuro. En este escenario la reciente Ley Orgánica para la Mejora de la Calidad Educativa (LOMCE) representa un nuevo intento por racionalizar el sistema educativo.

Por otro lado, el actual contexto de recesión económica impone una nueva definición de las prioridades del gasto público. Resulta evidente que una de las principales preocupaciones de la administración pública es el control del déficit público y el sistema educativo no escapa a este escenario. En este marco para las autoridades educativas resulta atractivo evaluar e indagar acerca de cuáles son las prácticas y políticas que conducirían a un aumento de la eficiencia del sistema, esto es, a una mejora de los resultados a partir de una mejor gestión de los recursos disponibles. Avanzar en esta línea resulta más interesante aún ya que la evidencia empírica disponible demuestra que mayores recursos (ratio profesores/alumno, salarios de los docentes, ordenadores/alumno o el gasto por estudiante) no aseguran per se mejores resultados académicos (Hanushek, 2003). La presencia de ineficiencias en un sistema educativo implica que es posible una mejora paretiana del mismo ya sea mediante la reducción de los recursos educativos sin disminuir los resultados académicos, ó alternativamente, incrementando los resultados académicos con los actuales recusos disponibles.

La LOMCE parece querer avanzar en la idea de evaluar la eficiencia educativa y para ello se propone en ella implementar pruebas externas de evaluación. Una vez realizadas estas pruebas de forma censal y consistente en el tiempo, la LOMCE afirma en la modificación 62 que "las Administraciones educativas publicarán los resultados obtenidos por los centros docentes considerados en relación con los factores socioeconómicos y socioculturales del contexto en que radiquen, de acuerdo con lo indicado en los artículos 140 y siguientes de esta ley orgánica y en los términos que el Gobierno establezca reglamentariamente. Las administraciones educativas aplicarán medidas correctoras a los centros sostenidos con fondos públicos que no alcancen los 
niveles adecuados". Por primera vez en una ley educativa, la medición sistemática de la eficiencia de los centros educativos es un objetivo explícito.

Una vez que los datos de inputs y outputs sean recogidos la siguiente tarea será medir la eficiencia educativa. Para ello existen fundamentalmente dos grupos de metodologías: las técnicas paramétricas y las no paramétricas. Si bien las primeras han cobrado mayor importancia en los últimos años (Perelman y Santín, 2011; CrespoCebada et al. 2012 y Crespo-Cebada et al. 2013), las técnicas no paramétricas han sido las más utilizadas para evaluar la eficiciencia educativa, ya que por su flexibilidad se adaptan mejor a la complejidad productiva del sector educativo. En particular, la técnica más utilizada ha sido el Análisis Envolvente de Datos propuesta por Charnes et al. (1978,1981) y Banker et al. (1984).

A partir de dichos trabajos seminales numerosas ampliaciones han sido propuestas y aplicadas para evaluar diversas políticas públicas. Un ejemplo de ello son los modelos semiparamétricos o modelos de dos etapas propuestos por Ray (1991) y McCarty y Yaisawarng (1993). Estos modelos consisten en estimar el nivel de eficiencia de cada centro educativo mediante un modelo DEA y luego, en una segunda etapa, regresar dichos índices sobre diversas variables contextuales con el objetivo de indagar e identificar las fuentes de las ineficiencias. Por su sencillez y por el interés de sus resultados estos modelos han sido muy aplicados en los últimos veinte años en diferentes sectores y en el sector educativo (Mancebón y Molinero 2000; Afonso y St. Aubyn 2006; Cordero et al. 2008; St. Aubyn et al. 2009; Alexander y Jaforullah 2010; Crespo et al. 2010; De Jorge y Santín 2010). Sin embargo, Xue y Harker (1999) señalaron que los índices de eficiencia estimados no son independientes entre sí y por tanto las estimaciones que resultan en la segunda etapa estarían sesgadas. Simar y Wilson $(2007,2011)$ proponen incorporar bootstrap para salvar este inconveniente y así obtener estimaciones insesgadas .

Por otro lado, la gran mayoría de los estudios de medición de eficiencia educativa se han enfocado a la educación secundaria y universitaria, y son menos frecuentes las investigaciones disponibles en materia de educación primaria. Si bien a nivel internacional existen varios estudios (Grosskopf et al. 2001; Banker et al. 2004; Blackburn et al. 2013; Casalprim et al. 2013; Mancebón y Molinero 2000; Mizala et al. 2002; Thanassoulis 2002), para el caso español, no hemos encontrado antecedentes hasta el momento. Por tanto, consideramos que resulta de interés analizar el grado de 
‘Quiero cambiar a mi hijo de grupo!’. Factores explicativos de la eficiencia técnica de los colegios en España

eficiencia de los colegios de primaria y sus posibles causas ya que es a edades tempranas cuando sería más adecuado identificar los centros más eficientes con el fin de aprender sus métodos y poder extender las prácticas docentes exitosas a otros centros para aumentar la eficiencia del sistema.

En definitiva, el objetivo de la presente investigación es doble. En primer lugar se plantea la evaluación del nivel de eficiencia de la educación primaria en España. En segundo lugar, se propone identificar qué prácticas y políticas educativas podrían contribuir a corregir las ineficiencias encontradas en el sistema. De este modo, se pretende aportar nuevos elementos al actual debate nacional acerca de cómo medir y mejorar la eficiencia del sistema educativo español. Con este propósito, se utilizan los datos de la Evaluación General de Diagnóstico 2009 (EGD) del Ministerio de Educación, Cultura y Deporte.

El trabajo se organiza de la siguiente forma. En el apartado siguiente se presenta la metodología implementada en el estudio. En el tercer apartado se describe brevemente la EGD y las variables seleccionadas en la investigación. La cuarta sección está dedicada a los resultados obtenidos. Por último, en el apartado final se exhiben las principales conclusiones del estudio y se discuten las implicaciones que éstas podrían tener sobre el diseño de las políticas educativas.

\section{Metodología}

\subsection{La función de producción educativa.}

El concepto de función de producción hace referencia a la relación que existe entre inputs y outputs, dada una tecnología productiva. El enfoque teórico utilizado en el presente trabajo que relaciona los recursos escolares con los resultados educativos está basado en la función de producción educativa propuesta por Levin (1974), Hanushek (1979) y más recientemente por Hanushek et al. (2012) que se expresa en la Ecuación 1.

$$
A_{i}=f\left(B_{i}, S_{i}\right) \quad \text { Ecuación } 1
$$

Donde el subíndice $i$ se refiere al centro educativo. $A_{i}$ representa el vector de outputs educativos, en general asociados al promedio de los resultados académicos obtenidos por los alumnos en pruebas objetivas. Por otra parte, los inputs educativos se 
dividen en: $B_{i}$, que representa las características socioeconómicas medias de los hogares de los que provienen los alumnos; aproximadas normalmente por el nivel educativo y profesional de sus padres y el vector $S_{i}$ que hace referencia a los recursos educativos del centro.

La estimación de dicha función de producción educativa es llevada a cabo frecuentemente considerando la posible existencia de comportamientos ineficientes en los centros. En este caso, se estimaría una frontera productiva basada en los centros que maximizan sus resultados a partir de los inputs que utilizan. La medida de la ineficiencia sería por tanto, la distancia de cada centro a la frontera construida. La ineficiencia puede asociarse a diversos factores: incorrecta organización y/o gestión de los centros (Nechyba 2000; Woessman 2001), inadecuadas prácticas educativas que tienen lugar en el aula, métodos de evaluación inapropiados, escasa motivación e incentivos de profesores y/o alumnos, etc. Por tanto, asumiendo comportamientos ineficientes la función de producción a estimar vendría dada por la Ecuación 2:

$$
A_{i}=f\left(B_{i}, S_{i}\right)-u_{i} \quad \text { Ecuación } 2
$$

Donde $u_{i}$ representa el nivel de eficiencia. Valores nulos de $u_{i}$ suponen que las unidades analizadas son plenamente eficientes, es decir, dado su nivel de inputs educativos y dada la tecnología existente estos centros están maximizando y gestionando correctamente el nivel de outputs que obtienen. Valores positivos de $u_{i}$ señalarían que el centro es ineficiente y por tanto la cuantía de la ineficiencia indica la cantidad de output que podría ser aumentado si se eliminara la ineficiencia.

En suma, en el proceso productivo intervienen tres tipos de variables: los outputs educativos $\left(A_{i}\right)$, los inputs educativos $\left(B_{i}, S_{i}\right)$, y el nivel de eficiencia de cada centro educativo $\left(u_{i}\right)$. Una vez que los outputs y los inputs educativos están definidos es posible estimar la eficiencia de los centros educativos e identificar los principales factores que la explican. Para ello emplearemos la metodología adoptada de dos etapas, la cual se describe detalladamente a continuación. 


\subsection{Primera etapa: La Medición de la eficiencia mediante análisis envolvente de datos}

La estimación empírica de la eficiencia está asociada al concepto farreliano de eficiencia técnica (Farrel, 1957), basado en cómo construir la frontera productiva a partir de datos reales. La frontera productiva se define como el máximo nivel de output que una unidad (DMU) puede obtener dados los recursos y tecnología disponibles (modelo orientado al output). En la práctica dicha frontera no es observable, por tanto la estimación de la misma debe realizarse de forma empírica en base a las mejores prácticas (yardstick competition) de la muestra analizada.

Existen básicamente dos grandes grupos de técnicas para estimar dicha frontera: las técnicas paramétricas o econométricas y las no paramétricas, basadas en modelos de optimización matemática. Si bien en las últimas décadas se ha incrementado la utilización de las primeras (Perelman y Santín 2011a, 2011b; Crespo et al. 2013), las últimas han sido considerablemente más aplicadas para medir la eficiencia técnica educativa (Worthington 2001; Mancebón y Muñiz 2003) y en particular dentro de éstas, el Análisis Envolvente de Datos (DEA).

Como se ha mencionado en el apartado anterior, el modelo DEA ha sido utilizado ampliamente para la medición de la eficiencia en diversas áreas de gasto público desde los trabajos pioneros de Charnes, Cooper y Rhodes $(1978,1981)$ y Banker, Charnes y Cooper (1984). El principal motivo de su extensa aplicación radica en la gran flexibilidad de la técnica que permite adaptarse a las peculiaridades de la producción pública; fundamentalmente al carácter multidimensional del output, al desconocimiento de la tecnología de producción y a la falta de información acerca de los precios de las variables implicadas. La técnica consiste en obtener, mediante un problema de optimización lineal, una frontera de producción que incluya a todas las unidades eficientes, junto con sus combinaciones lineales. Por tanto, el índice de eficiencia estimado para cada unidad es una medida relativa que depende del desempeño del resto de unidades4. La formulación analítica del Modelo DEA orientado al output con rendimientos variables a escala (Modelo DEA-BCC) para cada unidad analizada es mostrada en la ecuación 3:

\footnotetext{
${ }^{4}$ Cabe destacar que la verdadera tecnología de producción es desconocida y por tanto la frontera estimada con DEA es definida a partir de las mejores prácticas observadas en la muestra analizada.
} 
$\theta_{i}^{D E A}=\max _{\lambda, \theta}\left\{\theta_{i} \mid \theta y_{i} \leq Y \lambda ; x_{i} \geq X \lambda ; n 1^{\prime} \lambda=1 ; \lambda \geq 0 ; \forall i=1, \ldots, n\right\} \quad$ Ecuación 3

Donde, para la i-ésima unidad: $\theta_{i} \geq 1$ es la medida de eficiencia, $y_{i}$ es el vector $(q \times 1)$ de outputs y $x_{i}$ es el vector $(p \times 1)$ de inputs. Y por tanto, X y Y son las respectivas matrices de inputs $(p \times n)$ y outputs $(q \times n)$. El vector $\lambda$ de dimensión $(n \times 1)$, contiene las ponderaciones óptimas para maximizar el desempeño de cada unidad determinados por la solución del problema. Cuando $\theta_{i}=1$ significa que la unidad es eficiente y se encuentra sobre la frontera, mientras que valores de $\theta_{i}>1$ implican que la unidad es ineficiente, siendo $\theta_{i}$ la distancia que existe entre la i-ésima unidad analizada y la frontera productiva. Por tanto, a mayor valor del índice de eficiencia $\theta_{i}$, mayor ineficiencia. Así por ejemplo, un valor de $\theta_{i}=1,2$ sugiere que la iésima unidad es ineficiente, y que si fuera eficiente podría obtener un 20\% más de todos sus outputs dados sus recursos disponibles.

\subsection{Segunda etapa: Factores determinantes de la eficiencia}

A partir de los los índices de eficiencia estimados, $\theta_{i}$, en una segunda etapa éstos se regresan sobre un vector $Z=\left(z_{1}, z_{2}, \ldots, z_{k}\right)$ de variables contextuales de los centros educativos, las cuales no están directamente relacionadas con la producción educativa pero sí pueden influir sobre el proceso de aprendizaje:

$$
\boldsymbol{\theta}_{\boldsymbol{i}}=\boldsymbol{f}\left(\boldsymbol{Z}_{i}, \boldsymbol{\beta}_{i}\right) \quad \text { Ecuación } 4
$$

El método más utilizado en la literatura para estimar la Ecuación 4 en esta segunda etapa es el modelo de regresión censurada y en algunos casos mínimos cuadrados ordinarios (MCO)5; del cual se puede extraer cuáles son los factores que explican los índices de eficiencia estimados:

$$
\widehat{\boldsymbol{\theta}}_{\boldsymbol{i}}=\boldsymbol{Z}_{\boldsymbol{i}} \widehat{\boldsymbol{\beta}}_{\boldsymbol{i}}+\boldsymbol{\varepsilon}_{\boldsymbol{i}} \quad \text { Ecuación } 5
$$

Estos modelos de regresión convencionales popularizados a primeros de los noventa por Ray (1991) y McCarty y Yaisawarng (1993) aplicados en la segunda etapa, han sido criticados en los últimos años argumentando que las estimaciones de los índices de eficiencia estimados en la primera etapa $\left(\widehat{\theta}_{l}\right)$ están correlacionados serialmente (Xue y Harker, 1999). Esto significa que los errores de la Ecuación 5 no

\footnotetext{
5 Para ver una revisión detallada véase Simar y Wilson (2007).
} 
‘Quiero cambiar a mi hijo de grupo!'. Factores explicativos de la eficiencia técnica de los colegios en España

cumplen con el supuesto de independencia, y por tanto las estimaciones que resulten estarán sesgadas. Esta correlación serial entre los índices de eficiencia surge por construcción, ya que como fue mencionado anteriormente los índices de eficiencia son medidas relativas y dependen del valor de otras variables. Simar y Wilson $(2007,2011)$ agregan otra posible fuente de sesgo en las estimaciones debido a la posible existencia de correlación entre el vector de variables contextuales $Z_{i}$ y el término de error $\varepsilon_{i}$.

Para salvar dichos inconvenientes, Simar and Wilson $(2007,2011)$ proponen dos algoritmos que incorporan la técnica de bootstrap al modelo de regresión truncada estándar. A continuación se presenta el Algoritmo \# 1 propuesto por Simar y Wilson (2007) que utilizaremos en la presente investigación para medir el impacto que sobre la eficiencia estimada poseen diversas variables asociadas a los centros educativos y a los alumnos ${ }^{6}$.

\section{$\underline{\text { Algoritmo \# } 1}$}

1) Estimar los índices de eficiencia $\hat{\delta}_{i} \forall i=1, \ldots, n$ mediante la resolución de la Ecuación 3.

2)Estimar $\hat{\beta} y \hat{\sigma}_{\varepsilon}$ mediante el método de máxima verosimilitud en una regresión truncada de $\hat{\delta}_{i}$ sobre $\mathrm{z}_{\mathrm{i}}$ (Ecuación 5), utilizando $m<n$ observaciones tal que $\hat{\delta}_{i}>1$.

3)Repetir los pasos (3.a - 3.c) $L^{7}$ veces mediante un bucle, obteniendo las estimaciones bootstrap de $\beta$ y $\sigma_{\varepsilon}$ :

a) Para cada $i=1, \ldots, m$ extraer $\varepsilon_{i}$ de una distribución normal $N\left(0, \hat{\sigma}_{\varepsilon}^{2}\right)$ truncada a la izquierda en $\left(1-z_{i} \hat{\beta}\right)$.

b) Nuevamente, para $i=1, \ldots, m$ estimar $\delta_{i}^{*}=z_{i} \hat{\beta}+\varepsilon_{i}$.

c) Utilizando máxima verosimilitud estimar la regresión truncada de $\delta_{i}^{*}$ sobre $z_{i}$, obteniendo las estimaciones $\hat{\beta}^{*} y \hat{\sigma}_{\varepsilon}{ }^{*}$.

Mediante experimientos Monte Carlo Simar y Wilson (2007) examinan el desempeño de ambos algoritmos en comparación con los resultados que se obtienen al aplicar un modelo Tobit y una regresión truncada bajo métodos convencionales de regresión, es decir, sin aplicar bootstrap. Los resultados obtenidos ponen de manifiesto

\footnotetext{
${ }^{6}$ Los autores proponen un Algoritmo simple \#1 y un Algoritmo doble \#2. La diferencia radica en que el segundo incorpora un bootstrap adicional en la primera etapa, que corrige las estimaciones de los índices de eficiencia. Su desempeño real en simulaciones es muy similar por lo que por simplicidad en este trabajo aplicamos el algoritmo 1.

7 Siguiendo a Simar y Wilson (2007) se define $L=2000$.
} 
la invalidez de las estimaciones del modelo Tobit bajo el proceso generador de datos del estudio, y a su vez, demuestran que ambos algoritmos ajustan mejor que los modelos de regresión truncada convencional.

\section{Datos y variables}

\subsection{Las Evaluaciones Generales de Diagnóstico}

Las evaluaciones generales de diagnóstico son llevadas a cabo por el Instituto de Evaluación Educativa (INEE) y los organismos correspondientes de las administraciones educativas españolas con el objetivo de obtener datos representativos a nivel nacional del grado de adquisición de las competencias básicas del currículo en enseñanza primaria y secundaria. Estas competencias básicas se asocian con contenidos curriculares que relacionan conocimientos, habilidades y actitudes transferibles y útiles para hacer frente a situaciones y problemas que se presentan en la vida real. En definitiva, se trata de valorar en qué medida la escuela prepara para la vida y forma a los estudiantes para asumir su papel como ciudadanos en una sociedad moderna. Las dos últimas evaluaciones se realizaron a alumnos en cuarto curso de Educación Primaria en el año 2009 y a alumnos en segundo curso de Educación Secundaria Obligatoria en 2010. Las EGD proporcionan además de los resultados académicos un enorme volumen de información acerca de los alumnos y su familia, de los profesores y directores de los centros educativos, lo cual permite incorporar al análisis del rendimiento académico diversas variables contextuales y factores relacionados con los procesos organizativos y/o de aula.

En este trabajo se analiza la eficiencia de la educación primaria y por tanto se llevará a cabo en base a la información proveniente de la EGD 2009. La misma proporciona información a nivel de clase o distintos grupos de alumnos dentro del mismo colegio, a diferencia de la mayor parte de los estudios internacionales y nacionales disponibles hasta el momento que sólo proporcionan información a nivel de centros educativos. Este es un aspecto original y muy interesante, ya que en aquellos centros para los que se dispone información de dos grupos será posible comparar el nivel de eficiencia de ambos grupos. De este modo podemos obtener una medida que ilustre como las posibles diferencias entre profesores debidas a métodos de trabajo distintos dentro del aula impactan sobre los resultados académicos de los alumnos. 
‘Quiero cambiar a mi hijo de grupo!'. Factores explicativos de la eficiencia técnica de los colegios en España

En la EGD 2009 participaron 28.708 alumnos distribuidos en 1.358 grupos en 887 centros educativos españoles ${ }^{8}$. Teniendo en cuenta los objetivos del presente trabajo y con el fin de comparar unidades homogéneas se eliminaron del análisis los centros de enseñanza privada financiados exclusivamente con recursos privados y los grupos con menos de 10 alumnos. Se consideran entonces en el presente estudio 1.272 grupos de cuarto curso de primaria, de los cuales el $31 \%$ corresponden a centros concertados y el $69 \%$ a colegios públicos.

\subsection{Outputs, inputs y variables explicativas de la eficiencia.}

Para llevar a cabo el estudio se seleccionaron tres tipos de variables: los outputs e inputs utilizados en la primera etapa para estimar los índices de eficiencia, y por otra parte, las variables explicativas de dichos índices a incluir en la segunda etapa.

\section{Outputs e inputs}

La medición empírica de la educación recibida por un individuo es muy difícil de llevar a cabo, sobre todo cuando se desea analizar la calidad de la misma más allá de cuantificar los años de estudio. Existe un amplio consenso en la literatura acerca de considerar como outputs educativos los resultados que surjan de pruebas objetivas, como lo son las pruebas EGD 2009, ya que son difíciles de falsificar y sobre todo, son tomadas en cuenta por padres y políticos en el momento de evaluar el producto educativo para la toma de decisiones (Hoxby, 1999). En efecto, Hanushek (1997) observa que dos tercios de los estudios utilizan los resultados en las pruebas como medida de los outputs educativos. Por tanto, en el presente estudio, se seleccionan como outputs dos variables: el resultado medio obtenido en cada grupo en comunicación lingüística y matemática ("Lectura” y “Matemáticas” respectivamente).

En cuanto a los inputs del proceso educativo, se seleccionaron cuatro variables teniendo en cuenta la función de producción educativa presentada anteriormente

\footnotetext{
${ }^{8}$ La muestra fue extraída a partir del alumnado matriculado en cuarto año de educación primaria en el año 2009 utilizándose un muestreo bietápico estratificado y por conglomerados. Las diferentes CCAA definen los estratos y los centros educativos y, en su caso, los grupos dentro de éstos definen los conglomerados.

9 No ha sido considerado el resultado medio en las restantes dos competencias básicas (“Conocimiento e interacción con el mundo físico" y "Sociedad y ciudadana”) dado que aportan escasa información adicional debido a la alta correlación que presentan con los resultados medios incluidos en el análisis. Como fue mencionado, el modelo DEA pierde capacidad de discriminación a medida que se incrementan las dimensiones, y por tanto se priorizó la parsimonia seleccionando sólo dos outputs.
} 
(Ecuación 1), y que a su vez representan los insumos mínimos necesarios para llevar a cabo el proceso de aprendizaje (materia prima, capital físico y capital humano). A su vez, en el momento de la selección de la cantidad de inputs a incluir, se priorizó la parsimonia del modelo, con el propósito de mantener un buen nivel de discriminación del mismo. Los inputs seleccionados son los siguientes:

- Índice de estatus social, económico y cultural (ISEC): es un índice elaborado por los analistas de la EGD y que refleja el contexto social y familiar del alumno, y por tanto tiene en cuenta "la materia prima" a transformar en el proceso de aprendizaje. El mismo surge de aplicar un Análisis Factorial teniendo en cuenta cuatro componentes ${ }^{10}$ : el nivel más alto de estudio de los padres, la profesión más alta de los padres, el número de libros en el domicilio familiar y el nivel de recursos domésticos.

- Porcentaje de alumnos nativos en el grupo (PNAT): varios estudios en España han demostrado que el hecho de ser inmigrante afecta negativamente sobre los resultados académicos esperados de los alumnos (Calero et al. 2009; Salinas y Santín 2012; Zinovyeva et al. 2013). Por tanto, el porcentaje de nativos en el grupo evaluado debe ser considerado a la hora de comparar los resultados de distintos grupos para que sea una comparación justa, ya que caracteriza la materia prima del proceso productivo al igual que el ISEC.

- Porcentaje de alumnos sin dificultades de aprendizaje en el grupo (PNODIFAP): al igual que las variables anteriores, esta variable refleja una característica fundamental del alumnado a ser atendido por el profesor.

- Índice de calidad de los recursos educativos (ICRE): es un indicador que representa la calidad de los recursos educativos en el centro de enseñanza, y que se asocia por tanto al capital disponible en el centro educativo. El mismo es resultado de llevar a cabo un Análisis Factorial a partir de la respuesta de los profesores a cuatro preguntas relacionadas con la escasez o inadecuación de: material de enseñanza, ordenadores para la enseñanza, personal de apoyo docente, y por último, otro personal de apoyo. A mayor valor del índice, menor escasez y por tanto mejor calidad de los recursos educativos del centro. ${ }^{10}$ El ISEC está expresado como un valor tipificado para el promedio de España con media cero y desviación
típica 1. 
‘Quiero cambiar a mi hijo de grupo!'. Factores explicativos de la eficiencia técnica de los colegios en España

Para garantizar una adecuada especificación del modelo DEA, es necesario verificar que se cumpla el supuesto de monotonicidad de todos los inputs. Este supuesto garantiza que la tecnología productiva es tal que más de un input supone igual o más output. A continuación se presentan las correlaciones bivariadas de outputs e inputs, donde se verifica una correlación positiva y significativa en todos los inputs del modelo planteado.

Tabla 1. Correlaciones bivariadas Outputs-Inputs.

\begin{tabular}{lcccc}
\hline Out $/$ Inp & ISEC & PNAT & PNODIFAP & ICRE \\
\hline Matemáticas & $0,648^{* *}$ & $0,162^{* *}$ & $0,187^{* *}$ & $0,099^{* *}$ \\
Lectura & $0,680^{* *}$ & $0,202^{* *}$ & $0,227^{* *}$ & $0,085^{* *}$ \\
\hline$* *$ Correlaciones significativas al nivel 0.01 &
\end{tabular}

Cabe la pena mencionar que dada la información disponible en la EGD no se ha podido introducir ningún input relacionado con la calidad de los maestros del centro. Esta es una variable muy compleja y difícilmente cuantificable. La única variable disponible al respecto es el nivel de formación del profesor, y particularmente en el caso español la formación de los maestros es muy homogénea y por tanto, esta variable no permite discriminar los buenos de los malos docentes. Podría incluirse sí la cantidad de maestros. Sin embargo, el ratio de maestros cada 100 alumnos presenta correlación negativa y significativa con los resultados académicos, lo cual contradice la condición de monotonicidad previamente comentada. Adicionalmente, aunque existe cierta evidencia en diseños experimentales de que el tamaño del aula puede influir positivamente en los resultado académicos (Angrist y Lavy, 1999), cuando esta variable no es exógena los padres demandan las mejores escuelas y por tanto sus aulas aumentan de tamaño distorsionando su efecto sobre los resultados ${ }^{11}$.

\section{Variables explicativas de la eficiencia}

En cuanto a las variables explicativas de la ineficiencia que se utilizan en la Ecuación 4 en la segunda etapa, se seleccionaron diversas variables asociadas a las

\footnotetext{
${ }^{11}$ De todas formas, a efectos de verificar la robustez del modelo especificado, se realizó la estimación de los índices de eficiencia incluyendo esta variable como input, y no se encontraron diferencias con el modelo que excluye el ratio profesor/alumno. Por tanto, al no aportar información adicional como input y presentar correlación negativa con los outputs educativos se decidió excluirla del análisis DEA.
} 
características de los alumnos, de los centros educativos evaluados y otras variables que reflejan aspectos relacionados con la gestión y organización escolar y los procesos de enseñanza-aprendizaje llevados a cabo en las aulas.

Se seleccionaron las siguientes variables asociadas a las características del alumnado y del centro educativo:

- Titularidad (TITULAR): variable dicotómica que toma valor uno cuando el centro evaluado es público. Resulta especialmente interesante contrastar si el hecho de ser un centro público o privado impacta en el nivel de eficiencia, ya que este punto es uno de los focos de atención en los debates educativos en diversos países. Si bien a nivel internacional no existe aún concenso acerca de su impacto real, para el caso de España varios estudios que han estudiado la influencia de la titularidad del colegio sobre la eficiencia educativa sin resultados claros (Perelman y Santín 2011a, 2011b; Cordero et al. 2011; Mancebón et al. 2012 y Crespo et al. 2013).

- Centro ubicado en una localidad con 500.0oo o más habitantes (GRANLOC): variable dicotómica que toma valor uno cuando el centro educativo está ubicado en una localidad de más de 500.00o habitantes.

- Porcentaje de alumnas en el grupo (PMUJER): se pretende analizar si la composición del grupo según género afecta a la eficiencia del mismo.

- Porcentaje de alumnos repetidores en el grupo (PREPITE): esta variable refleja la política de repetición adoptada por cada centro. Es una de las variables claves en el debate educativo a la hora de analizar la calidad de la enseñanza, ya que se asocia fuertemente al fracaso escolar, uno de los principales problemas del sistema educativo español.

- Porcentaje de alumnos en el grupo que estudian entre una y dos horas diarias (PESTUDIA): variable que surge de la respuesta de los alumnos a la pregunta cuánto tiempo dedica a estudiar después del colegio. Se espera a priori un impacto positivo en la eficiencia.

- Porcentaje de alumnos en el grupo que lee diariamente (PLEE): al igual que la variable anterior surge de la respuesta de los alumnos a la pregunta con qué frecuencia leen fuera del colegio. Varios estudios internacionales han demostrado que la lectura extracurricular tiene un impacto positivo en los resultados académicos 
‘Quiero cambiar a mi hijo de grupo!’. Factores explicativos de la eficiencia técnica de los colegios en España

(Machin y McNally, 2004). En el presente trabajo se quiere contrastar esta hipótesis para el caso español.

- Porcentaje de alumnos en el grupo que recibe ayuda de la familia para realizar los deberes (PAYUDA): surge de la respuesta de los alumnos que dicen recibir ayuda de sus padres o abuelos para realizar los deberes en su casa. Existe un amplio consenso acerca de la importancia que tiene el involucramientos de la familia en el proceso de aprendizaje de sus hijos, y de la importancia que tiene brindarles apoyo a lo largo de todo el ciclo escolar. Nuestra hipótesis de partida es que esta variable debería tener un efecto positivo sobre la eficiencia educativa.

- Asistencia de muchos o bastantes padres a entrevistas con profesores (PADENT): variable dicotómica que toma valor uno cuando el director del centro responde que "Muchos" o "Bastantes" padres suelen acudir a entrevistas con los profesores en el centro evaluado. $\mathrm{Al}$ igual que la variable anterior, la asistencia a reuniones refleja el interés y el apoyo que los padres tienen respecto a la educación de sus hijos. Es de esperar que esta variable afecte de forma positiva a la eficiencia ya que los padres ejercerían una mayor presión sobre el centro y sus docentes.

Adicionalmente, se seleccionaron algunas variables que caracterizan a los directores y a los profesores del grupo evaluado. Existe controversia acerca del verdadero impacto de estas variables sobre los resultados académicos de los alumnos, por tanto no esperamos ningún impacto específico a priori.

- Director con menos de 5 años de experiencia (DIREXP5): variable dicotómica que toma valor uno cuando el director del centro posee menos de 5 años de experiencia como director.

- Director con 20 o más años de experiencia (DIREXP2O): variable dicotómica que toma valor uno cuando el director del centro posee 20 años o más de experiencia como director.

- Género del profesor (PROFMUJ): variable dicotómica que toma valor uno cuando el profesor del grupo es mujer (Mora y Escardibul (2013)).

- Profesor con experiencia docente menor a 5 años (PROFEXP5): variable dicotómica que toma valor uno cuando el profesor del curso posee menos de 5 años de experiencia docente. 
- Profesor con 30 años o más de experiencia docente (PROFEXP3O): variable dicotómica que toma valor uno cuando el profesor del curso posee 30 años o más de experiencia docente.

En los útlimos años diversas investigaciones han analizado el impacto que tiene sobre los resultados agrupar al alumnado según sus habilidades. Sin embargo, aún no existe evidencia concluyente acerca del verdadero efecto y para el caso español, no existen estudios previos específicos que analicen este aspecto. Hanushek y Woessmann (2006) analizan el impacto de la agrupación prematura (a los 10 años de edad) para diversos países de la OECD y encuentran que la misma incrementa la desigualdad entre los resultados de los estudiantes; sin embargo, no encuentran un efecto significativo sobre los resultados en sí mismos. Por tanto, se incluyen en la presente investigación diversas variables que reflejan la agrupación del alumnado a nivel de primaria de España para medir su efecto sobre la eficiencia de los grupos evaluados.

- Agrupación de alumnos en el centro según rendimiento académico (AGREND): variable dicotómica que toma valor uno cuando en el centro educativo evaluado se agrupa a los estudiantes según su rendimiento académico.

- Agrupación de alumnos en el centro buscando homogeneidad en los grupos (AGRHOMO): variable dicotómica que toma valor uno cuando en el centro educativo evaluado se agrupa a los estudiantes buscando homogeneidad dentro del grupo.

- Agrupación de alumnos en el centro buscando heterogeneidad en los grupos (AGRHETE): variable dicotómica que toma valor uno cuando en el centro educativo evaluado se agrupa a los estudiantes buscando heterogeneidad dentro del grupo.

Por último, se consideran en el análisis diversas variables asociadas a la forma de trabajo dentro del aula. Existe actualmente cierto debate acerca de cuáles son las técnicas de aprendizaje más efectivas, si aquellas de estilo "tradicional" o por el contrario las llamadas prácticas "modernas". Recientemente, Lavy (2011) en un trabajo donde mide la relación entre técnicas de enseñanza-aprendizaje en clase y los resultados académicos de los estudiantes, encuentra que el tipo de técnica tiene un impacto muy significativo sobre los resultados y que el éxito de una u otras depende del tipo de alumno de cada clase (género, habilidades, etc.). Por tanto, incorporamos al 
‘Quiero cambiar a mi hijo de grupo!’. Factores explicativos de la eficiencia técnica de los colegios en España

análisis ciertas variables que reflejan la forma de trabajo dentro de la clase con el fin de medir su impacto en la eficiencia del grupo evaluado.

- El profesor explica la mayor parte de la clase (PEXP): porcentaje de alumnos que responde "Siempre" o "Casi siempre" cuando se les pregunta con qué frecuencia el profesor explica la mayor parte del tiempo de la clase.

- Los alumnos realizan los ejercicios propuestos en clase (PEJER): porcentaje de alumnos que responde "Siempre" o "Casi siempre" cuando se les pregunta con qué frecuencia los alumnos hacen los ejercicios propuestos por los profesores en clase.

- Trabajo individual de los alumnos en clase (PINDIV): porcentaje de alumnos que responde "Siempre" o "Casi siempre" cuando se le pregunta con qué frecuencia los alumnos trabajan individualmente en clase.

En la Tabla 2 se presentan los principales estadísticos descriptivos de los outputs, inputs y variables explicativas de la eficiencia. Los resultados se muestran para todos los centros analizados, y según titularidad de los mismos. Del análisis de la tabla se desprende que existe una importante heterogeneidad en los resultados, en especial cuando se comparan los centros públicos y concertados. A priori, se puede observar que estos últimos presentan mejores resultados académicos en ambas pruebas así como también mayor dotación inicial de recursos educativos. En la mayoría de los casos, se verifica una mayor homogeneidad de estas variables entre centros privados que entre centros públicos.

En cuanto a las variables explicativas de la eficiencia, en general los centros concertados presentan mejores valores medios que los centros públicos, aunque las diferencias a priori son más moderadas que en el caso de las observadas en los inputs y outputs del proceso educativo. Este hecho indicaría por tanto, que los mejores resultados académicos de los centros concertados podrían responder a una mayor dotación inicial de recursos y no a una mejor gestión de los mismos. 
Tabla 2. Estadísticos descriptivos de outputs, inputs y variables explicativas de la eficiencia.

\begin{tabular}{|c|c|c|c|c|c|c|c|c|c|c|c|c|}
\hline \multirow{2}{*}{ Variables } & \multicolumn{4}{|c|}{ Total de colegios } & \multicolumn{4}{|c|}{ Colegios concertados } & \multicolumn{4}{|c|}{ Colegios públicos } \\
\hline & Media & Desv.Est. & Min & Máx. & Media & Desv.Est. & Min & Máx. & Media & Desv.Est. & Min & Máx. \\
\hline \multicolumn{13}{|l|}{ Outputs } \\
\hline Matemáticas & 501,2 & 43,8 & 336,4 & 629,7 & 520,8 & 38,4 & 403,4 & 629,4 & 492,4 & 43,2 & 336,4 & 629,7 \\
\hline Lectura & 500,8 & 45,0 & 303,7 & 608,3 & 522,4 & 36,7 & 405,4 & 608,3 & 491,2 & 45,1 & 303,7 & 606,7 \\
\hline \multicolumn{13}{|l|}{ Inputs } \\
\hline ISEC & 1,78 & 0,55 & o & 3,03 & 2,20 & 0,44 & 0,69 & 3,03 & 1,59 & 0,49 & o & 2,90 \\
\hline PNAT & 0,89 & 0,13 & 0,17 & 1 & 0,94 & 0,09 & 0,28 & 1 & 0,87 & 0,15 & 0,17 & 1 \\
\hline PNODIFAP & 0,93 & 0,67 & 0,63 & 1 & 0,94 & 0,06 & 0,63 & 1 & 0,92 & 0,07 & 0,63 & 1 \\
\hline ICRE & 2,48 & 0,88 & 0 & 3,76 & 2,55 & 0,82 & o & 3,76 & 2,45 & 0,91 & o & 3,76 \\
\hline \multicolumn{13}{|c|}{ Variables explicativas } \\
\hline TITULAR & 0,69 & 0,46 & o & 1 & 0,00 & 0,00 & o & o & 1,00 & 0,00 & 1 & 1 \\
\hline GRANLOC & 0,09 & 0,29 & $\mathrm{O}$ & 1 & 0,16 & 0,37 & $\mathrm{O}$ & 1 & 0,06 & 0,24 & $\mathrm{O}$ & 1 \\
\hline PMUJER & 0,49 & 0,12 & o & 1 & 0,49 & 0,13 & o & 1 & 0,49 & 0,11 & 0,10 & 1 \\
\hline PREPITE & 0,08 & 0,08 & o & 0,60 & 0,05 & 0,06 & o & 0,38 & 0,09 & 0,09 & 0 & 0,60 \\
\hline PESTUDIA & 0,34 & 0,15 & $\mathrm{O}$ & 0,79 & 0,36 & 0,15 & 0,04 & 0,75 & 0,34 & 0,15 & $\mathrm{o}$ & 0,79 \\
\hline PLEE & 0,44 & 0,16 & o & 0,96 & 0,46 & 0,15 & 0,06 & 0,96 & 0,43 & 0,15 & o & 0,89 \\
\hline PAYUDA & 0,58 & 0,16 & 0,05 & 1 & 0,63 & 0,15 & 0,11 & 1 & 0,56 & 0,16 & 0,05 & 1 \\
\hline PADENT & 0,43 & 0,49 & o & 1 & 0,64 & 0,48 & 0 & 1 & 0,33 & 0,47 & o & 1 \\
\hline DIREXP $_{5}$ & 0,34 & 0,47 & o & 1 & 0,31 & 0,46 & $\mathrm{O}$ & 1 & 0,35 & 0,48 & $\mathrm{O}$ & 1 \\
\hline DIREXP2O & 0,12 & 0,32 & o & 1 & 0,14 & 0,34 & o & 1 & 0,11 & 0,31 & o & 1 \\
\hline AGREND & 0,09 & 0,29 & o & 1 & 0,10 & 0,31 & 0 & 1 & 0,09 & 0,28 & o & 1 \\
\hline AGRHOMO & 0,14 & 0,35 & o & 1 & 0,17 & 0,38 & $\mathrm{O}$ & 1 & 0,12 & 0,33 & $\mathrm{O}$ & 1 \\
\hline AGRHETE & 0,50 & 0,50 & o & 1 & 0,44 & 0,50 & o & 1 & 0,53 & 0,50 & o & 1 \\
\hline PROFMUJ & 0,74 & 0,44 & o & 1 & 0,72 & 0,45 & o & 1 & 0,75 & 0,43 & o & 1 \\
\hline PROFEXP5 & 0,10 & 0,30 & o & 1 & 0,11 & 0,32 & o & 1 & 0,10 & 0,30 & o & 1 \\
\hline PROFEXP3O & 0,35 & 0,48 & $\mathrm{O}$ & 1 & 0,32 & 0,47 & $\mathrm{O}$ & 1 & 0,37 & 0,48 & $\mathrm{O}$ & 1 \\
\hline PEXP & 0,49 & 0,17 & o & 1 & 0,45 & 0,17 & o & 0,88 & 0,51 & 0,17 & o & 1 \\
\hline PEJER & 0,88 & 0,09 & 0,33 & 1 & 0,90 & 0,09 & 0,39 & 1 & 0,88 & 0,09 & 0,33 & 1 \\
\hline PINDIV & 0,75 & 0,15 & 0 & 1 & 0,78 & 0,15 & 0 & 1 & 0,74 & 0,15 & 0,10 & 1 \\
\hline
\end{tabular}




\section{Resultados}

A continuación se presenta la distribución de los índices de eficiencia $\widehat{\theta}_{l}$, estimados a partir del modelo DEA orientado al output y con rendimientos variables a escala definido en la Ecuación 3. Los resultados señalan que sólo el 5\% de los grupos evaluados son eficientes (presentan un índice de eficiencia igual a uno). El índice de eficiencia promedio de los colegios evaluados asciende a 1,12, lo cual significa que en promedio, los centros evaluados podrían incrementar sus resultados en matemáticas y lectura en un 12\% utilizando los recursos actuales. Más aún, dada la dotación inicial de inputs, casi un $45 \%$ podría incrementarlos entre un $10 \%$ y un $20 \%$, y un $15 \%$ de los grupos podrían mejorar sus resultados académicos entre un $20 \%$ y un $40 \%$ si fueran eficientes.

Figura 1. Distribución de Índices de Eficiencia (DEA-BBC).

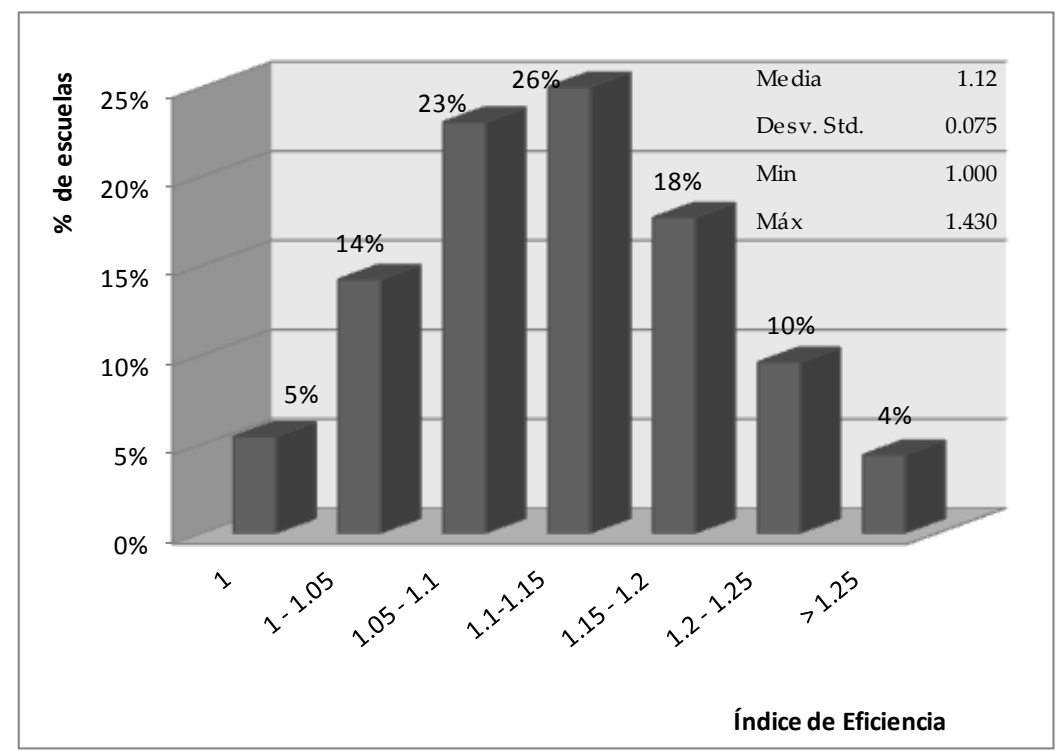

Una vez estimados los índices de eficiencia, los mismos se regresan sobre diversas variables contextuales referentes a los alumnos y a los centros educativos evaluados. La Tabla 3 muestra los resultados de las estimaciones de segunda etapa (Ecuación 5).

En primer lugar, en cuanto a las características del centro educativo se observa que la titularidad pública tiene un efecto positivo y significativo sobre la eficiencia estimada. Por tanto, los mejores resultados académicos observados en los centros privados se corresponden con una mayor dotación inicial de recursos pero no con una 
gestión más eficiente de los mismos. Este es un resultado interesante en el debate actual acerca de si incidir o no en trasladar la gestión de algunos centros públicos de enseñanza primaria a la órbita privada. En este sentido los resultados evidencian que la gestión privada no asegura una mejora en la eficiencia educativa al menos a nivel de educación primaria. Sin embargo, los resultados deben ser interpretados con cautela, ya que es en esta investigación se analiza la eficiencia técnica y no se tiene en cuenta el coste monetario de los recursos (eficiencia asignativa). En general, el mayor coste educativo que enfrentan los centros de enseñanza está asociado a la contratación del personal docente y en España éste es significativamente mayor en los colegios públicos que privados, por tanto este factor también debe ser considerado a la hora de evaluar la conveniencia de uno y otro tipo de gestión.

Tabla 3. Resultados de las estimaciones de la segunda etapa.

\begin{tabular}{|c|c|c|c|c|}
\hline $\begin{array}{l}\text { Variable } \\
\text { dependiente: } \\
\text { Nivel de eficiencia }\end{array}$ & Coef. & $\begin{array}{l}\text { Error Est. } \\
\text { Bootstrap }\end{array}$ & $\mathrm{P}>|\mathrm{Z}|$ & $\begin{array}{c}\text { Efecto } \\
\text { marginal }\end{array}$ \\
\hline TITULAR ${ }^{* *}$ & $-0,011$ & 0,005 & 0,042 & $-0,008$ \\
\hline GRANLOC $* *$ & $-0,027$ & 0,009 & 0,002 & $-0,020$ \\
\hline PMUJER & 0,022 & 0,020 & 0,268 & 0,017 \\
\hline PREPITE ** & 0,109 & 0,034 & 0,001 & 0,085 \\
\hline PESTUDIA** & $-0,135$ & 0,017 & 0,000 & $-0,105$ \\
\hline $\mathrm{PLEE}^{* *}$ & $-0,068$ & 0,017 & 0,000 & $-0,053$ \\
\hline PAYUDA** & $-0,052$ & 0,016 & 0,001 & $-0,041$ \\
\hline PADENT** & $-0,014$ & 0,005 & 0,003 & $-0,011$ \\
\hline $\operatorname{DIREXP}_{5}$ & $-0,004$ & 0,005 & 0,482 & $-0,003$ \\
\hline DIREXP2O & $-0,007$ & 0,008 & 0,401 & $-0,005$ \\
\hline AGREND & $-0,01$ & 0,008 & 0,227 & $-0,008$ \\
\hline AGRHOMO & $-0,001$ & 0,007 & o,897 & $-0,001$ \\
\hline AGRHETE & 0,003 & 0,005 & 0,519 & 0,002 \\
\hline PROFMUJ* & $-0,01$ & 0,005 & 0,066 & $-0,008$ \\
\hline PROFEXP5 & $-0,005$ & 0,008 & 0,546 & $-0,004$ \\
\hline PROFEXP $30^{* *}$ & $-0,014$ & 0,005 & 0,008 & $-0,011$ \\
\hline PEXP ** & 0,066 & 0,015 & 0,000 & 0,052 \\
\hline PEJER** & $-0,097$ & 0,027 & 0,000 & $-0,076$ \\
\hline PINDIV ${ }^{* *}$ & $-0,093$ & 0,016 & o,000 & $-0,073$ \\
\hline constante & 1,356 & 0,026 & 0,000 & --- \\
\hline /sigma & 0,071 & 0,002 & & \\
\hline
\end{tabular}


Asimismo, el hecho de que el centro se ubique en grandes ciudades (500.000 o más habitantes) donde existe una mayor oferta de centros educativos, impacta positivamente en la eficiencia de los centros quizás debido a que al haber una mayor presión por competir por los alumnos los centros necesitan ofrecer una buena gestión.

Por el contrario, las variables asociadas a la experiencia de los directores del centro no presentan efectos significativos sobre los resultados académicos de los estudiantes. Tampoco presentan coeficientes significativos las variables que denotan la agrupación del alumnado según distintos factores.

En cuanto a las características del alumnado, se observa que el género de los alumnos no afecta a la eficiencia del grupo evaluado. Por el contrario, la proporción de alumnos repetidores tiene un impacto negativo y muy fuerte. Este resultado, conduce a cuestionarse acerca de la conveniencia de las políticas actuales de repetición de cursos del sistema educativo. Sería por tanto tal vez más adecuado, intentar en edades más tempranas, detectar a los alumnos con mayor riesgo de repetición y brindarles un apoyo adicional con el fin de evitar que efectivamente repitan el curso.

La variable que más afecta a la eficiencia está asociada al tiempo que dedican los alumnos a realizar los deberes del colegio en casa. Así, dedicar entre una y dos horas diarias a estudiar después del colegio mejora la eficiencia del grupo. Este es un resultado que presenta desafíos importantes a la hora de diseñar las políticas educativas, ya que no sólo depende de la voluntad y trabajo de los docentes y colegios sino que además requiere del compromiso e implicación de las familias fuera del horario escolar. Otra variable que tiene un fuerte efecto positivo sobre la eficiencia es la lectura extracurricular. De este modo, los grupos con mayor proporción de alumnos que leen diariamente resultan ser más eficientes. Por tanto, si se desea incrementar la eficiencia educativa, sería apropiado llevar a cabo acciones para incentivar a los alumnos a la lectura extracurricular tanto desde el centro educativo como desde la propia familia. Este resultado debe ser interpretado con cautela, ya que el tiempo dedicado a la lectura es extracurricular y por tanto en principio no implica sustituir las horas necesarias para cumplir con las tareas académicas cotidianas, sino que debe ser un complemento de las mismas. Adicionalmente, los grupos donde mayor proporción de alumnos reciben ayuda de su familia para realizar los deberes se muestran más eficientes así como los grupos donde la mayor parte de los padres asiste a reuniones o entrevistas con los profesores. Al igual que lo comentado anteriormente, la implicación 
y apoyo de la familia en el proceso de aprendizaje de los alumnos es fundamental para obtener buenos resultados y no es posible sustituirlos. En este sentido, será necesario lograr mayor compromiso de las familias en aquellos casos donde se observen estas carencias y a su vez, en los casos en que no sea posible lograr este compromiso brindar apoyo adicional a estos estudiantes para compensar en cierta medida dichas carencias familiares.

En cuanto a las características del profesorado y las prácticas de enseñanza dentro del aula, ser profesora y poseer más de treinta años de experiencia impactan positivamente aunque de forma débil sobre la eficiencia; mientras que por el contrario, ser un profesor inexperto (tener menos de 5 años de experiencia docente) no tiene efectos sobre la misma. Los factores que sí parecen impactar sobre la eficiencia del grupo de forma significativa son las relacionadas con la forma de trabajo dentro del aula. Así, sería recomendable para incrementar la eficiencia en clase que el profesor evite explicar la mayor parte del tiempo de la clase, incentivar a los alumnos a que efectivamente realicen los ejercicios que proponen los docentes y promover el trabajo individual dentro del aula ${ }^{12}$.

\section{Diferencia de eficiencia entre grupos en el mismo colegio}

Como fue mencionado anteriormente, las estimaciones se han realizado a nivel de grupos lo cual permite comparar el nivel de eficiencia entre grupos en aquellos centros donde se evaluaron dos grupos. En estos casos, las características del centro y del alumnado son muy similares en ambos grupos, y por tanto las diferencias en el nivel de eficiencia responden básicamente a un efecto fijo explicado por las diferentes características del profesorado y de las prácticas llevadas a cabo dentro del aula. Este tipo de análisis aporta por tanto una nueva perspectiva complementaria a los modelos de dos etapas a la hora de intentar explicar las ineficiencias educativas. En los últimos años numerosas investigaciones han demostrado que el valor que agregan los profesores mediante su motivación y el trabajo que realizan en las aulas es un componente esencial a la hora de explicar el rendimiento de los estudiantes (Rivkin et al. 2010). En este sentido, la comparación del nivel de eficiencia entre grupos de un mismo centro nos permitiría identificar cuáles son las prácticas y técnicas docentes

${ }^{12}$ Los resultados obtenidos en la segunda etapa deben ser interpretados con cautela, ya que únicamente permiten explorar cuáles son las posibles causas de la eficiencia de los centros educativos. Para demostrar la verdadera causalidad de estas variables sería necesario llevar a cabo un diseño experimental que permita controlar la posible existencia de endogeneidad de alguna de estas variables. 
llevadas a cabo dentro del aula que contribuirían a alcanzar mejores resultados educativos. Esto es, identificar las prácticas docentes que contribuyan a incrementar la eficiencia educativa de los grupos más ineficientes (benchmarking).

Tabla 4. Diferencias absolutas en el nivel de eficiencia intergrupal.

\begin{tabular}{lc}
\hline $\begin{array}{l}\text { Diferencias en } \\
\text { eficiencia }\end{array}$ & \% de centros \\
\hline [0- 0,05$)$ & 51 \\
[0,05- 0,10] & 35,5 \\
+0,10 & 13,5 \\
\hline $\begin{array}{l}\text { Nota: Las diferencias fueron calculadas } \\
\text { como la diferencia absoluta entre el índice } \\
\text { de eficiencia de los dos grupos del centro } \\
\text { educativo evaluado. }\end{array}$
\end{tabular}

La Tabla 4 presenta la distribución de los centros evaluados con dos grupos según la diferencia absoluta en el nivel de eficiencia de estos grupos. De ella se desprende, que en la mitad de los centros evaluados las diferencias de eficiencia entre los dos grupos del mismo centro son menores a 0,05 puntos, lo cual implica que existe en estos centros cierta homogeneidad y coordinación por parte de los docentes en las técnicas aplicadas en ambos grupos. Por el contrario, en un 35,5\% de los centros las diferencias de eficiencia entre los dos grupos evaluados son entre 0,05 y 0,10 puntos. Esto es, en estos centros, uno de los grupos podría incrementar entre un $5 \%$ y un $10 \%$ sus resultados educativos si fuera igual de eficiente que el otro grupo. Finalmente, un 13,5\% de los centros presenta diferencias mayores a 0,10. A modo de ejemplo, el centro donde se encuentran mayores diferencias entre los dos grupos evaluados $(0,43)$, presenta un índice de eficiencia estimado de 1 y 1,43 respectivamente para cada grupo. Esto significa, que en un mismo centro educativo donde ambos grupos poseen similar composición del alumnado, un grupo es técnicamente eficiente mientras que el otro podría mejorar sus resultados en un $43 \%$. Sería por tanto interesante, en una futura investigación poder analizar con mayor profundidad aquellos casos donde se observan mayores discrepancias, con el fin de identificar cuáles son las prácticas dentro del aula que conducen a los mejores resultados de modo de obtener un sistema educativo más eficiente. Es también importante avanzar en la comparación entre grupos dentro de un colegio no sólo por motivos de eficiencia sino también de equidad, ya que sería 
necesario que los padres y los centros controlaran que los alumnos tendrán oportunidades de aprendizaje similares independientemente del grupo al que asistan.

\section{Comentarios finales}

La presencia de ineficiencias en un sistema educativo implica que podrían incrementarse los resultados obtenidos dados los recursos disponibles en el sistema, lo cual resulta ser una de las principales preocupaciones de los gobiernos actuales. Si la meta de largo plazo es disponer en un futuro de una población con mayor y mejor nivel de estudios que garantice un crecimiento económico basado en una alta productividad del factor trabajo cualificado, es imprescindible disponer de un sistema educativo más eficiente. En este sentido, el presente estudio busca analizar si existen comportamientos ineficientes en los centros de educación primaria en España y a su vez, identificar potenciales políticas y prácticas educativas que contribuyan a mejorar la eficiencia en los centros ineficientes. Para cumplir con dicho propósito, se ha llevado a cabo un modelo semi-paramétrico en dos etapas basado en la información de la EGD 2009.

Los resultados corroboran que en educación primaria existen ya comportamientos ineficientes no despreciables y que por tanto, es posible incrementar la eficiencia si se adoptan las políticas educativas adecuadas, tanto desde el diseño de las mismas por parte de las autoridades educativas, como desde la implementación de éstas por parte de los colegios. Los centros evaluados podrían incrementar en media los resultados obtenidos en un $12 \%$ dada su dotación actual de recursos educativos. En efecto, casi un $15 \%$ centros podría incrementar más de un 20\% sus resultados, y un $45 \%$ podría incrementarlos entre un 10\% y un 20\% dada su dotación inicial de recursos.

En este sentido, los resultados de la segunda etapa conducen a identificar conclusiones muy atractivas desde el punto de vista de la planificación y ejecución de las políticas y prácticas educativas. En primer lugar, los centros de titularidad pública parecen ser más eficientes en promedio que los privados concertados, lo cual indica que los mejores resultados medios obtenidos por estos últimos responden a una mayor dotación incial de recursos y a un entorno para el aprendizaje más favorable y no a una mejor gestión. Este resultado estaría indicando que trasladar la gestión de los centros 
de enseñanza primaria públicos a la órbita privada no garantiza mejoras en la eficiencia técnica educativa.

En segundo lugar, sería adecuado identificar a edades tempranas aquellos alumnos con mayor riesgo de repetición y brindarles apoyo adicional para evitar que efectivamente repitan, ya que este hecho impacta negativamente en sus resultados. Asimismo, leer diariamente, dedicar entre una y dos horas diarias a hacer los deberes y recibir ayuda de la familia para hacerlos incrementa la eficiencia educativa, así como el hecho de que los padres asistan a entrevistas con los profesores de sus hijos. Por tanto, resulta fundamental promover y alcanzar un mayor compromiso, motivación y apoyo de las familias en el proceso de aprendizaje de sus hijos, sobre todo en edades más tempranas que es cuando éstos más lo perciben y lo necesitan. En cuanto al trabajo dentro del aula, evitar que el profesor explique la mayor parte del tiempo de la clase, incentivar a los alumnos a que efectivamente realicen los ejercicios que proponen los docentes en clase y promover el trabajo individual dentro del aula parecen ser técnicas de enseñanza-aprendizaje que contribuyen a incrementar la eficiencia educativa.

Por último, el presente estudio permite por primera vez comparar la eficiencia entre grupos de educación primaria de un mismo centro y de este modo dar cuenta del aporte que cada profesor y el trabajo dentro del aula tienen sobre los resultados educativos de sus estudiantes. Los resultados indican que una vez se controla por el tipo de alumno, el contexto y el colegio, existen diferencias significativas en la eficiencia educativa entre las aulas de un mismo centro. Este hecho, pone de manifiesto la necesidad de profundizar en este tipo de análisis para entender qué ocurre dentro de cada aula e identificar en un futuro, mediante técnicas cuantitativas y cualitativas, cuáles son las prácticas educativas llevadas a cabo en los grupos más eficientes. 


\section{Referencias}

Afonso, A., \& St Aubyn, M. (2006). Cross-country efficiency of secondary education provision: A semi-parametric analysis with non-discretionary inputs. Economic modelling, 23(3), 476-491.

Alexander, W. R. J., Haug, A. A., \& Jaforullah, M. (2010). A two-stage double-bootstrap data envelopment analysis of efficiency differences of New Zealand secondary schools. Journal of Productivity Analysis, 34(2), 99-110.

Angrist, J.D. \& Lavy, V. (1999). Using Maimonides' rule to estimate the effect of class size on scholastic achievement. The Quarterly Journal of Economics 114 (2), 533575 .

Banker, R. D., Charnes, A., \& Cooper, W. W. (1984). Some models for estimating technical and scale inefficiencies in data envelopment analysis. Management Science, 3o(9), 1078-1092.

Banker, R. D., Janakiraman, S., \& Natarajan, R. (2004). Analysis of trends in technical and allocative efficiency: An application to Texas public school districts. European Journal of Operational Research, 154(2), 477-491.

Barro, R. J. (2001). Human capital and growth. The American Economic Review, 91(2), 12-17.

Blackburn, V., Brennan, S., \& Ruggiero, J. (2013). Measuring efficiency in Australian Schools: A preliminary analysis. Socio-Economic Planning Sciences.

Calero Martínez, J., Choi de Mendizábal, A., \& Waisgrais, S. (2009). Determinantes del rendimiento educativo del alumnado de origen nacional e inmigrante en PISA-2006. Cuadernos económicos de ICE(78), 281-310.

Casalprim, M., Rialp, J., Prior, D., \& Sabrià, B. (2013). An Assessment of Schools' Efficiency of Different Educational Systems. Mediterranean Journal of Social Sciences, 4(11), 631.

Cordero-Ferrera, J. M., Pedraja-Chaparro, F., \& Salinas-Jiménez, J. (2008). Measuring efficiency in education: an analysis of different approaches for incorporating nondiscretionary inputs. Applied Economics, 4O(10), 1323-1339.

Cordero Ferrera, J. M., Crespo Cebada, E., \& Santín González, D. (2010). Factors affecting educational attainment: evidence from Spanish PISA 2006 Results. Regional and Sectoral Economic Studies, 10(3). 
Crespo-Cebada, E., Pedraja-Chaparro, F., \& Santín, D. (2013). Does school ownership matter? An unbiased efficiency comparison for regions of Spain. Journal of Productivity Analysis. doi: 10.1007/s11123-013-0338-y

Charnes, A., Cooper, W., Lewin, A. Y., \& Seiford, L. M. (1997). Data envelopment analysis theory, methodology and applications. Journal of the Operational Research Society, 48(3), 332-333.

Charnes, A., Cooper, W. W., \& Rhodes, E. (1978). Measuring the efficiency of decision making units. European Journal of Operational Research, 2(6), 429-444.

Charnes, A., Cooper, W. W., \& Rhodes, E. (1981). Evaluating program and managerial efficiency: an application of data envelopment analysis to program follow through. Management Science, 27(6), 668-697.

De La Fuente, A. (2011). Human capital and productivity. Nordic Economic Policy Review, 2, 103-131.

Escardíbul, J.-O., \& Mora, T. (2013). Teacher Gender and Student Performance in Mathematics. Evidence from Catalonia (Spain). Journal of Education and Training Studies, 1(1), p39-46.

Farrell, M. J. (1957). The measurement of productive efficiency. Journal of the Royal Statistical Society. Series A (General), 120(3), 253-290.

Grosskopf, S., Hayes, K. J., Taylor, L. L., \& Weber, W. L. (2001). On the determinants of school district efficiency: Competition and monitoring. Journal of Urban Economics, 49(3), 453-478.

Hanushek, E. A. (1979). Conceptual and empirical issues in the estimation of educational production functions. Journal of human Resources, 351-388.

Hanushek, E. A. (1997). Assessing the effects of school resources on student performance: An update. Educational evaluation and policy analysis, 19(2), 141164.

Hanushek, E. A. (2003). The Failure of Input-based Schooling Policies*. The Economic Journal, 113(485), F64-F98.

Hanushek, E. A. (2006). Does Educational Tracking Affect Performance and Inequality? Differences-in-Differences Evidence Across Countries*. The Economic Journal, 116(510), C63-C76.

Hanushek, E. A., \& Kimko, D. D. (2000). Schooling, labor-force quality, and the growth of nations. American economic review, 1184-1208. 
Hanushek, E. A., Link, S., \& Woessmann, L. (2012). Does school autonomy make sense everywhere? Panel estimates from PISA. Journal of Development Economics.

Hanushek, E. A., \& Woessmann, L. (2006). Does early tracking affect educational inequality and performance? Differences-in-differences evidence across countries. Economic Journal, 116(510), C63-C76.

Hanushek, E. A., \& Woessmann, L. (2008). The role of cognitive skills in economic development. Journal of economic literature, 607-668.

Hoxby, C. M. (1999). The productivity of schools and other local public goods producers. Journal of Public Economics, 74(1), 1-30.

Lavy, V. (2011). What makes an effective teacher? Quasi-experimental evidence. National Bureau of Economic Research, No. w16885.

Levin, H. M. (1974). Measuring efficiency in educational production. Public Finance Quarterly, 2(1), 3-24.

Machin, S., \& McNally, S. (2008). The literacy hour. Journal of Public Economics, 92(5), 1441-1462.

Mancebón, M. J., Calero, J., Choi, Á., \& Ximénez-de-Embún, D. P. (2012). The efficiency of public and publicly subsidized high schools in Spain: Evidence from PISA-2006. Journal of the Operational Research Society, 63(11), 1516-1533.

Mancebón, M. J., \& Molinero, C. M. (2000). Performance in primary schools. Journal of the Operational Research Society, 51(7), 843-854.

McCarty, T. A., \& Yaisawarng, S. (1993). Technical efficiency in New Jersey school districts. The measurement of productive efficiency: Techniques and applications, 271-287.

Mizala, A., Romaguera, P., \& Farren, D. (2002). The technical efficiency of schools in Chile. Applied Economics, 34(12), 1533-1552.

Moreno, J., \& Santín González, D. (2010). Determinantes de la eficiencia educativa en la Unión Europea. Hacienda pública española(193), 131-155.

Muñiz, M. A., \& Mancebón, M. J. (2003). Aspectos clave de la evaluación de la eficiencia productiva en la educación secundaria. Papeles de Economía Española(95), 162-187.

Perelman, S., \& Santin, D. (2011a). Imposing monotonicity on outputs in parametric distance function estimations. Applied Economics, 43(30), 4651-4661.

Perelman, S., \& Santin, D. (2011b). Measuring educational efficiency at student level with parametric stochastic distance functions: an application to Spanish PISA results. Education Economics, 19(1), 29-49. 
Ray, S. C. (1991). Resource-use efficiency in public schools: A study of Connecticut data. Management Science, 37(12), 1620-1628.

Rivkin, S. G., Hanushek, E. A., \& Kain, J. F. (2005). Teachers, schools, and academic achievement. Econometrica, 73(2), 417-458.

Salinas, J., \& Santín, D. (2012). Selección escolar y efectos de la inmigración sobre los resultados académicos españoles en PISA 2006. Revista de educación, 358, 382405 .

Simar, L., \& Wilson, P. W. (2007). Estimation and inference in two-stage, semiparametric models of production processes. Journal of econometrics, 136(1), 31-64.

Simar, L., \& Wilson, P. W. (2011). Two-stage DEA: caveat emptor. Journal of Productivity Analysis, 36(2), 205-218.

St. Aubyn, M., Garcia, F., \& Pais, J. (2009). Study on the efficiency and effectiveness of public spending on tertiary education. Economic Papers 390, European Commission.

Thanassoulis, E., Da Conceição, M., \& Silva Portela, A. (2002). School Outcomes: Sharing the Responsibility Between Pupil and School1. Education Economics, 1O(2), $183-207$.

Worthington, A. C. (2001). An empirical survey of frontier efficiency measurement techniques in education. Education Economics, 9(3), 245-268.

Xue, M., \& Harker, P. T. (1999). Overcoming the inherent dependency of DEA efficiency scores: a bootstrap approach. Unpublished Working Paper, Wharton Financial Institutions Center, University of Pennsylvania.

Zinovyeva, N., Felgueroso, F., \& Vazquez, P. (2013). Immigration and student achievement in Spain: evidence from PISA. Journal of the Spanish Economic Association, , SERIEs. doi: 10.1007/s13209-013-0101-7. 